\title{
COVID-19 en el paciente en diálisis. Una revisión sistemática
}

\author{
Lucía Parra-Martos ${ }^{1}$, Olga Cobacho-Salmoral ${ }^{1}$, Julia Martínez-Urbano ${ }^{1}$, Rodolfo Crespo-Montero ${ }^{1,2,3}$ \\ ${ }^{1}$ Departamento de Enfermería. Facultad de Medicina y Enfermería. Universidad de Córdoba. España \\ ${ }^{2}$ Servicio de Nefrología. Hospital Universitario Reina Sofía. Córdoba. España \\ ${ }^{3}$ Instituto Maimónides de Investigación Biomédica de Córdoba. España
}

\begin{abstract}
Como citar este artículo: Parra-Martos L, Cobacho-Salmoral 0, Martínez-Urbano J, Crespo-Montero R. COVID-19 en el paciente en diálisis. Una revisión sistemática. Enferm Nefrol. 2021 0ct-Dic;24(4):351-63
\end{abstract}

\section{Resumen}

Introducción: La pandemia por COVID-19 acaecida en 2019 afectó con mayor gravedad a la población de mayor edad y con enfermedades subyacentes, siendo estos factores de riesgo de mortalidad. Estas características son propias del paciente renal en diálisis, por lo que es importante conocer cuál fue el impacto del COVID-19 en este grupo poblacional.

Objetivo: El objetivo principal fue conocer y sintetizar la evidencia científica existente sobre las características clínicas de la enfermedad COVID-19 en el paciente con enfermedad renal crónica en diálisis.

Metodología: Se realizó una revisión sistemática basada en la declaración PRISMA, en la que se analizaron 23 artículos procedentes de las bases de datos PubMed y Scopus. Se incluyeron artículos originales en inglés y español que excluyeran la población pediátrica.

Resultados: Se incluyeron 23 artículos de diseño observacional. Principalmente los resultados se centraron en incidencia, características clínicas, como sintomatología o evolución, mortalidad y medidas preventivas en las unidades de diálisis.

\section{Correspondencia:}

Lucía Parra Martos

Email: lucia.pm18@gmail.com
Conclusiones: La incidencia de la COVID-19 fue mayor en unidades de diálisis que en los pacientes que recibían diálisis domiciliaria. La sintomatología más leve fue la común a la población general. En los casos más graves, cerca del $50 \%$ de los pacientes necesitaron hospitalización y/o ingreso en una Unidad de Cuidados Intensivos. La mortalidad fue bastante elevada y se relacionó con la edad avanzada, comorbilidad, sexo masculino o fragilidad clínica. Además, en las unidades de diálisis se implementaron medidas de prevención de la enfermedad, al ser espacios físicos cerrados donde se concentra un gran número de pacientes.

PALABRAS CLAVE: COVID-19; diálisis renal; hemodiálisis; diálisis peritoneal.

\section{COVID-19 in the dialysis patient. A systematic review}

\section{Abstract}

Introduction: The COVID-19 pandemic that occurred in 2019 most severely affected the older population and those with underlying diseases, these being risk factors for mortality. Circumstances present in renal patients on dialysis, so it is important to know what the impact of COVID-19 was on this population group.

Objective: To understand and synthesise the existing scientific evidence on the clinical characteristics of 
COVID-19 disease in patients with chronic kidney disease on dialysis.

Material and Method: A systematic review was carried out based on the PRISMA statement, in which 23 articles from the PubMed and Scopus databases were analysed. Original articles in English and Spanish that excluded the paediatric population were included.

Results: 23 articles of observational design were included. Mainly the results focused on incidence, clinical characteristics, such as symptomatology or evolution, mortality and preventive measures in dialysis units.

Conclusions: The incidence of COVID-19 was higher in dialysis units than in patients receiving home dialysis. The mildest symptomatology was common to the general population. In the most severe cases, about $50 \%$ of patients required hospitalisation and/or admission to an Intensive Care Unit. Mortality was quite high and was related to advanced age, comorbidity, male sex or clinical frailty. In addition, disease prevention measures were implemented in the dialysis units, as they are closed physical spaces where many patients are concentrated.

KEYWORDS: COVID-19; renal dialysis; hemodialysis; peritoneal dialysis.

\section{Introducción}

En diciembre de 2019 aparecieron varios casos de pacientes con enfermedad respiratoria aguda de origen desconocido en Wuhan, China. Tras varios estudios, se detectó que la enfermedad estaba causada por un nuevo tipo de betacoronavirus al que denominaron inicialmente 2019-nCoV ${ }^{1,2}$.

En los siguientes meses, tanto el número de infectados como de fallecidos aumentó bruscamente, llevando a la Organización Mundial de la Salud (OMS) a declarar el nuevo coronavirus como una pandemia mundial el día 11 de marzo de $2020^{3}$. Finalmente, la enfermedad producida por este nuevo virus fue denominada $\mathrm{CO}$ VID-19 mientras que el agente causal pasó de llamarse 2019-nCoV a SARS-CoV-2 ${ }^{4}$.

En un estudio llevado a cabo sobre 70.000 casos en China, la enfermedad se presentaba en el $87 \%$ de los casos en personas entre 30 y 79 años y más del $80 \%$ cursaron como infecciones leves ${ }^{5}$. Aunque en ese momento se desconocía esta entidad clínica, se comenza- ron a publicar los síntomas más frecuentes ${ }^{6}$, entre los que destacaban: fiebre, tos, fatiga, sensación de falta de aire, mialgias y dolor de cabeza. Otros síntomas como: congestión, dolor de garganta o expectoración y síntomas gastrointestinales.

Además, como complicación más frecuente se destacó la aparición de neumonía, que en los casos más graves puede desembocar en: síndrome de distrés respiratorio agudo (SDRA), fallo respiratorio con requerimiento de ventilación mecánica invasiva, shock y fallo multiorgánico, pudiendo cualquiera de ellas conducir a la muerte ${ }^{7}$.

De hecho, la tasa global de mortalidad en el estudio mencionado anteriormente alcanzó el 2,3\%5, aunque con notables diferencias según la edad de las personas: pacientes entre 70 y 79 años suponían el $8 \%$ de los fallecidos y los mayores de 80 años alcanzaban el porcentaje de $14,8 \%$. La población de edad más avanzada tuvo menor incidencia de casos, pero con una tasa de mortalidad muy alta. Además, los pacientes con más comorbilidades presentaron una mortalidad mayor, entre las que destacaban: enfermedades cardiovasculares, diabetes, enfermedad respiratoria crónica, hipertensión o cáncer. Por lo tanto, la combinación de edad avanzada y presencia de comorbilidades fue un factor de riesgo de mortalidad en la población general ${ }^{5}$.

Por otra parte, la Enfermedad Renal Crónica (ERC) representa un importante problema de salud pública, tanto por la elevada incidencia y prevalencia, como por la morbimortalidad y el alto coste socioeconómico ${ }^{8}$. EI estudio EPIRCE describe que la ERC afecta aproximadamente al $10 \%$ de la población general adulta y a más de un $20 \%$ en personas mayores de 60 años 9 . En su última etapa o estadio 5, es necesario instaurar una terapia renal sustitutiva como tratamiento cróni$\mathrm{Co}^{10}$, como son el trasplante renal o un procedimiento de diálisis, que puede ser hemodiálisis (HD) o diálisis peritoneal (DP) ${ }^{11}$.

Por otro lado, la Sociedad Española de Nefrología, en su último informe sobre diálisis y trasplantes, describe el perfil de paciente en diálisis de la siguiente forma: edad mayor de 65 años, sexo masculino predominante, diabetes mellitus como causa principal de ERC ${ }^{12}$ y enfermedades cardiovasculares como la comorbilidad más frecuente (sobre todo cardiopatía isquémica, enfermedad vascular periférica e insuficiencia cardiaca congestiva) ${ }^{13}$.

Teniendo en cuenta este perfil del paciente en diálisis y, dado que en un principio la COVID 19 afectaba de 
forma más grave a la población general con algunos de estos factores de riesgo, nos pareció importante realizar esta revisión con el fin de conocer el impacto que esta enfermedad ha tenido en el paciente con ERC en diálisis.

\section{Objetivo principal}

El objetivo principal de este estudio fue conocer y sintetizar la evidencia científica existente acerca de la incidencia y las características clínicas de la enfermedad COVID-19 en pacientes en diálisis.

\section{Objetivos específicos.}

- Determinar la mortalidad y principales factores de riesgo de muerte en pacientes en diálisis por $\mathrm{CO}$ VID-19.

- Describir las medidas de prevención y control contra el COVID-19 llevadas a cabo en unidades de diálisis.

\section{Metodología}

\section{Diseño del estudio}

Se ha llevo a cabo una revisión sistemática de estudios indexados en bases de datos de ciencias de la salud. Se ha realizado una síntesis temática categorial de los artículos seleccionados.

La revisión sistemática se ha desarrollado en base a la declaración PRISMA (Preferred Reporting Items for Systematic Reviews and Meta-Analyses $)^{14}$ :

\section{Métodos de búsqueda}

Las bases de datos empleadas fueron PubMed y Scopus. La estrategia de búsqueda se estableció con los siguientes términos MeSH: "COVID-19", "dialysis", "renal dialysis", "hemodialysis" y "peritoneal dialysis".

Los términos se combinaron con los operadores booleanos "AND" y "OR", siendo la estrategia la siguiente: ("COVID-19" AND (("dialysis") OR ("renal dialysis") OR ("hemodialysis") OR ("peritoneal dialysis")). Con el operador "AND" se delimitaron los grandes bloques de búsqueda (COVID-19, dialysis) y con el operador "OR" en el interior de los paréntesis para incluir sinónimos u otros ítems relacionados.

\section{Criterios de inclusión y exclusión}

Los criterios de inclusión y exclusión establecidos fueron: Criterios de inclusión:

- Artículos originales publicados desde enero de 2020 a marzo de 2021.
- Artículos que trataran sobre la infección por C0VID-19 en pacientes en diálisis.

- Artículos en inglés y español.

Criterios de exclusión:

- Artículos sin texto completo disponible.

- Artículos sobre el tema en población pediátrica.

\section{Resultados de la búsqueda}

Una vez establecida la estrategia de búsqueda en las diferentes bases de datos seleccionadas, se procede a la búsqueda de artículos desde noviembre del año 2020 hasta abril de 2021 . El proceso de búsqueda de estudios se plasma en un diagrama de flujo PRISMA (figura 1).

Al incluir la estrategia de búsqueda en las bases de datos referidas se encontraron 254 artículos. Se eliminaron los manuscritos duplicados (15), quedando 239 para la lectura de título y resumen. Una vez revisado el título y resumen se eliminaron 183 manuscritos, quedando para lectura completa 56 artículos. Tras la lectura a texto completo, se excluyeron 33 artículos de los 56. Los motivos de exclusión se asociaron al diseño de estudio. Finalmente fueron 23 los artículos seleccionados.

\section{Evaluación de la calidad de los artículos}

La calidad de los artículos seleccionados se determinó de acuerdo con las listas de verificación establecidas por STROBE ${ }^{15}$. Además, se complementó con el índice de impacto de las revistas de las que fueron extraídos los artículos utilizados mediante Scimago Journal \& Country Rank $(S J R)^{16}$, elaborado a partir de la base de datos Scopus.

\section{Extracción de datos}

Se extrajeron las siguientes variables de los artículos seleccionados: autor, año, muestra, país, diseño metodológico del estudio, resultados principales y calidad de la evidencia.

\section{Síntesis de resultados}

Se seleccionaron 23 artículos para la presente revisión sistemática, según los criterios de inclusión y exclusión establecidos. Se realizó un enfoque temático-categorial debido a la existencia de heterogeneidad de resultados y variables analizadas en los artículos estudiados. 


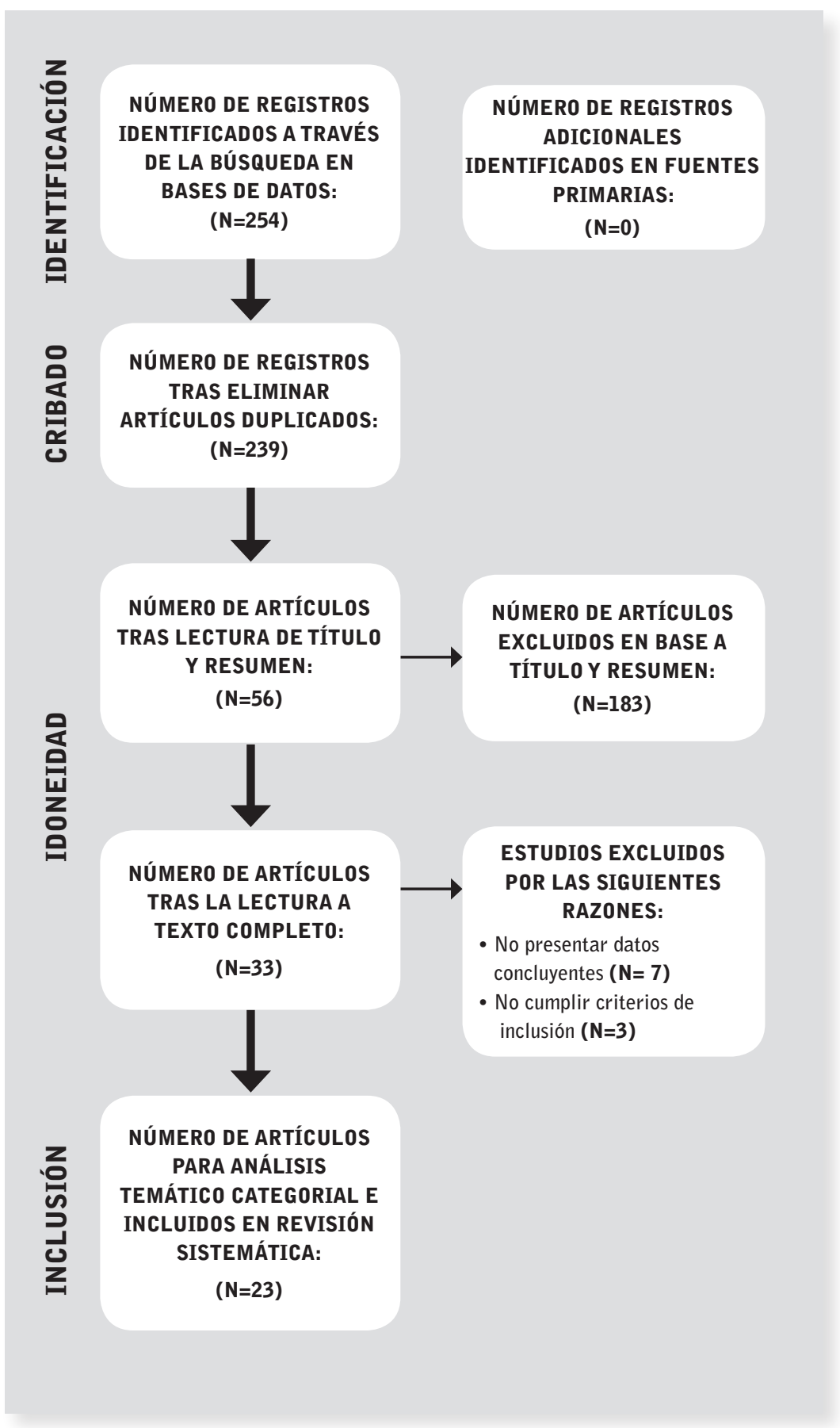

Figura 1. Diagrama de flujo del proceso de búsqueda.

\section{Resultados}

\section{Selección de los artículos}

Los 23 artículos seleccionados fueron de diseño observacional, 14 retrospectivos, 7 prospectivos, 1 descriptivo transversal y 1 de casos y controles. En la tabla 1 se muestran las principales características de los artículos seleccionados.

\section{Análisis de los resultados}

Incidencia y factores de riesgo de contagio De los 23 artículos analizados, 11 incluían pacientes de ambas modalidades de diálisis, HD y DP.

La incidencia se encontraba entre $1,5 \%$ y $5,5 \%$ en los estudios que incluían ambos tipos de pacientes, aunque el $90 \%$ de estos contagios fueron enfermos que recibían HD y en torno al $5 \%$ eran pacientes en $D P^{20,21,25,31}$. También hay que destacar que la proporción de pacientes en HD y DP incluidos en los estudios fue muy descompensada, ya que la mayoría de ellos recibían HD.

Por otro lado, otros estudios únicamente incluían pacientes de HD y, en este caso, su incidencia de COVID-19 fluctuó entre $7,4 \%$ y $15 \%{ }^{18,28,34,36,27}$. Uno de los estudios reflejaba una incidencia general (pacientes de HD y de DP) del $19,6 \%$ debido a que se realizó durante el primer brote de coronavirus que hubo en Wuhan en diciembre de $2019^{29}$.

Los factores de riesgo para contraer la infección por COVID-19 según los artículos analizados fueron: sexo masculino, recibir HD en centros sanitarios u hospitales, vivir en residencias de ancianos, usar transporte institucional colectivo para acudir a las sesiones de HD o recibir diálisis 3 veces a la semana $(87 \%)$ en lugar de 2 sesiones $^{20,23,25,27}$.

\section{Características clínicas de la enfermedad}

\section{- Síntomas $18,19,24,26,27,30$.}

Los tres síntomas más prevalentes en esta población fueron fiebre, disnea y tos. Otras dolencias como fatiga, mialgia, dolor de cabeza y de garganta, escalofríos o sintomatología gastrointestinal (diarrea, náuseas y vómitos) fueron muy comunes en los estudios analizados. La pérdida del sentido del gusto (ageusia) y del olfato (anosmia)17,21,36 también estuvo presente en parte de la población. En dos de los artículos revisados los pacientes también manifestaron algún tipo de alteración mental en el $25 \%^{31}$ y el $8 \%{ }^{35}$ de la población. 
Tabla 1. Resumen de la extracción de datos de los artículos.

\begin{tabular}{|c|c|c|c|c|c|c|}
\hline Autor & Tipo de estudio & Muestra & Objetivos & $\begin{array}{l}\text { Tipo } \\
\text { de diálisis }\end{array}$ & Conclusiones & $\begin{array}{l}\text { Evaluación } \\
\text { de calidad }\end{array}$ \\
\hline $\begin{array}{l}\text { Aoun M et } \\
\text { al }{ }^{17} \text {. Líbano, } \\
2021 .\end{array}$ & $\begin{array}{l}\text { Estudio } \\
\text { observacional } \\
\text { retrospectivo. }\end{array}$ & 231 & $\begin{array}{l}\text { Describir las } \\
\text { características de } \\
\text { pacientes en HD con } \\
\text { COVID-19 y analizar } \\
\text { los factores ligados a } \\
\text { mayor mortalidad. }\end{array}$ & HD & $\begin{array}{l}\text { Este estudio identificó que: mayor edad, presencia } \\
\text { de multimorbilidades, arteriopatía coronaria o } \\
\text { insuficiencia cardíaca, así como diagnóstico de } \\
\text { neumonía son factores de mal pronóstico. }\end{array}$ & $\begin{array}{l}\text { STROBE: } \\
21 / 22 \\
\text { SJR: Q2 }\end{array}$ \\
\hline $\begin{array}{l}\text { Alberici F et } \\
a{ }^{18} \text {. Italia, } \\
2020 .\end{array}$ & $\begin{array}{l}\text { Estudio } \\
\text { observacional } \\
\text { de cohortes } \\
\text { retrospectivo. }\end{array}$ & 94 & $\begin{array}{l}\text { Describir las } \\
\text { características y las } \\
\text { consecuencias en } \\
\text { pacientes de HD de } \\
\text { mantenimiento con } \\
\text { COVID-19. }\end{array}$ & HD & $\begin{array}{l}\text { Los datos mostraron las características de los } \\
\text { pacientes infectados: edad media de } 72 \text { años y } \\
\text { con al menos una comorbilidad. } 63 \% \text { del total } \\
\text { desarrollaron SDRA y el } 29 \% \text { fallecieron debido } \\
\text { a fallo respiratorio. }\end{array}$ & $\begin{array}{l}\text { STROBE: } \\
\text { 19/22 } \\
\text { SJR: Q1 }\end{array}$ \\
\hline $\begin{array}{l}\text { Xiong } F \text { et } \\
\text { a }{ }^{19} \text {. China, } \\
2020 .\end{array}$ & $\begin{array}{l}\text { Estudio } \\
\text { observacional } \\
\text { retrospectivo. }\end{array}$ & 131 & $\begin{array}{l}\text { Examinar los datos } \\
\text { de comorbilidad, } \\
\text { las características } \\
\text { clínicas de COVID-19 } \\
\text { en pacientes en HD } \\
\text { y la eficacia de las } \\
\text { medidas para el } \\
\text { control epidémico en } \\
\text { los centros de HD. }\end{array}$ & HD & $\begin{array}{l}\text { El } 95 \% \text { de los pacientes infectados tenían al } \\
\text { menos una comorbilidad. } \\
\text { Los síntomas principales fueron fiebre, } \\
\text { fatiga, tos, expectoración, disnea y síntomas } \\
\text { gastrointestinales. Un total de } 101 \text { pacientes } \\
\text { padecieron una infección leve/moderada y los } 30 \\
\text { restantes tuvieron una enfermedad severa/crítica. }\end{array}$ & $\begin{array}{l}\text { STROBE: } \\
22 / 22 \\
\text { SJR: Q1 }\end{array}$ \\
\hline $\begin{array}{l}\text { Hsu M.C et } \\
\text { aRo. Estados } \\
\text { Unidos, } \\
2021\end{array}$ & $\begin{array}{l}\text { Estudio } \\
\text { observacional } \\
\text { retrospectivo. }\end{array}$ & 438 & $\begin{array}{l}\text { Buscar y caracterizar } \\
\text { los factores de riesgo } \\
\text { y las consecuencias } \\
\text { posteriores al } \\
\text { COVID-19 en esta } \\
\text { población. }\end{array}$ & HD, DP & $\begin{array}{l}\text { Los resultados mostraron que el } 94 \% \text { de los } \\
\text { infectados recibían HD en centros urbanos y que } \\
\text { los pacientes que recibían diálisis domiciliaria } \\
\text { eran menos propensos a enfermar por COVID-19. }\end{array}$ & $\begin{array}{l}\text { STROBE: } \\
\text { 22/22 } \\
\text { SJR: Q1 }\end{array}$ \\
\hline $\begin{array}{l}\text { Lano G et } \\
a^{R 1} . \text { Francia, } \\
2020 .\end{array}$ & $\begin{array}{l}\text { Estudio } \\
\text { observacional de } \\
\text { cohortes. }\end{array}$ & 122 & $\begin{array}{l}\text { Determinar los } \\
\text { factores de riesgo } \\
\text { de una evolución } \\
\text { crítica y la mortalidad } \\
\text { en pacientes en } \\
\text { diálisis crónica con } \\
\text { COVID-19. }\end{array}$ & $H D, D P$ & $\begin{array}{l}\text { Los datos mostraron que el } 37 \% \text { tuvo una } \\
\text { evolución crítica: } 16 \% \text { ingresó en UCI y } \\
\text { el } 28 \% \text { falleció. Los factores de riesgo de } \\
\text { evolución crítica fueron: disnea y necesidad de } \\
\text { oxigenoterapia, un bajo recuento de linfocitos } \\
\text { al ingreso e historia de enfermedad vascular } \\
\text { periférica. }\end{array}$ & $\begin{array}{l}\text { STROBE: } \\
\text { 22/22 } \\
\text { SJR: Q1 }\end{array}$ \\
\hline $\begin{array}{l}\text { Jager JK, } \\
\text { et aR2. } \\
\text { Holanda, } \\
2020 \text {. }\end{array}$ & $\begin{array}{l}\text { Estudio } \\
\text { observacional } \\
\text { descriptivo. }\end{array}$ & 3.285 & $\begin{array}{l}\text { Estudiar la mortalidad } \\
28 \text { días después } \\
\text { del diagnóstico de } \\
\text { COVID-19 de la } \\
\text { población europea en } \\
\text { terapia de reemplazo } \\
\text { renal. }\end{array}$ & HD, DP & $\begin{array}{l}\text { Los resultados fueron: a los } 28 \text { días falleció el } \\
20 \% \text { de la población. } \\
\text { La mortalidad era mayor en pacientes con más } \\
\text { de } 75 \text { años, que tenían como enfermedad renal } \\
\text { primaria la hipertensión y sexo masculino. }\end{array}$ & $\begin{array}{l}\text { STROBE: } \\
\text { 20/22 } \\
\text { SJR: Q1 }\end{array}$ \\
\hline $\begin{array}{l}\text { Taji MPH } \\
L_{\text {, et ap3. }} \\
\text { Canadá, } \\
2021 .\end{array}$ & $\begin{array}{l}\text { Estudio } \\
\text { observacional } \\
\text { prospectivo. }\end{array}$ & 187 & $\begin{array}{l}\text { Describir la incidencia, } \\
\text { las consecuencias y los } \\
\text { factores de riesgo de } \\
\text { infección por SARS- } \\
\text { CoV-2 y medir los } \\
\text { resultados incluyendo } \\
\text { mortalidad. }\end{array}$ & HD, DP & $\begin{array}{l}\text { Los resultados mostraron una incidencia del } \\
1,5 \% \text {. Algunos predictores de riesgo de contraer } \\
\text { la infección fueron: recibir hemodiálisis en centro } \\
\text { sanitario o vivir en una residencia. El } 28,3 \% \\
\text { falleció, siendo el único factor de riesgo la edad } \\
\text { avanzada. }\end{array}$ & $\begin{array}{l}\text { STROBE: } \\
21 / 22 \\
\text { SJR: Q1 }\end{array}$ \\
\hline $\begin{array}{l}\text { Turgutalp } \\
\text { K, et aR4. } \\
\text { Turquía, } \\
2021 .\end{array}$ & $\begin{array}{l}\text { Estudio } \\
\text { observacional } \\
\text { retrospectivo. }\end{array}$ & 567 & $\begin{array}{l}\text { Describir las } \\
\text { características tanto } \\
\text { clínicas, radiológicas } \\
\text { y de laboratorio y } \\
\text { los determinantes } \\
\text { de mortalidad en un } \\
\text { grupo de pacientes en } \\
\text { HD hospitalizados por } \\
\text { COVID-19. }\end{array}$ & HD & $\begin{array}{l}\text { La clínica común fue: fiebre, disnea, tos, mialgias, } \\
\text { dolor de cabeza/garganta, diarrea. } \\
\text { La mortalidad se relacionaba con: mayor edad, } \\
\text { presentación severa/crítica de la enfermedad, } \\
\text { padecer insuficiencia cardiaca y otros indicadores } \\
\text { de laboratorio. }\end{array}$ & $\begin{array}{l}\text { STROBE: } \\
21 / 22 \\
\text { SJR: Q2 }\end{array}$ \\
\hline
\end{tabular}




\begin{tabular}{|c|c|c|c|c|c|c|}
\hline Autor & Tipo de estudio & Muestra & Objetivos & $\begin{array}{l}\text { Tipo } \\
\text { de diálisis }\end{array}$ & Conclusiones & $\begin{array}{l}\text { Evaluación } \\
\text { de calidad }\end{array}$ \\
\hline $\begin{array}{l}\text { Couchoud } \\
\text { C, et aR5. } \\
\text { Francia, } \\
2020 \text {. }\end{array}$ & $\begin{array}{l}\text { Estudio } \\
\text { observacional casos } \\
\text { y controles. }\end{array}$ & 1.621 & $\begin{array}{l}\text { Estudiar la incidencia } \\
\text { de COVID-19 en la } \\
\text { población francesa } \\
\text { en diálisis, el curso } \\
\text { de la enfermedad e } \\
\text { identificar los factores } \\
\text { de riesgo asociados a } \\
\text { mortalidad. }\end{array}$ & $H D, D P$ & $\begin{array}{l}\text { La incidencia de casos de infectados fue } \\
\text { del } 3,3 \% \text {. El curso de la enfermedad se } \\
\text { dividía en: infección leve, infección moderada } \\
\text { (hospitalización), e infección severa (UCI). Los } \\
\text { factores de riesgo de mortalidad son: mayor edad, } \\
\text { presencia de comorbilidades cardiovasculares y } \\
\text { fragilidad clínica. }\end{array}$ & $\begin{array}{l}\text { STROBE: } \\
21 / 22 \\
\text { SJR: Q1 }\end{array}$ \\
\hline $\begin{array}{l}\text { Tomacruz } \\
\text { DI, et aR6. } \\
\text { Filipinas, } \\
2021 .\end{array}$ & $\begin{array}{l}\text { Estudio } \\
\text { observacional } \\
\text { de cohortes } \\
\text { retrospectivo. }\end{array}$ & 68 & $\begin{array}{l}\text { Determinar los } \\
\text { perfiles clínicos y las } \\
\text { consecuencias a corto } \\
\text { plazo de pacientes en } \\
\text { HD ingresados por } \\
\text { COVID-19. }\end{array}$ & HD & $\begin{array}{l}\text { El estudio mostró que los síntomas principales } \\
\text { fueron: disnea, fiebre, tos, fatiga, mialgia o } \\
\text { diarrea. La mortalidad estaba asociada con } \\
\text { insuficiencia respiratoria aguda, medida por bajo } \\
\text { cociente PF y necesidad de ventilación mecánica } \\
\text { invasiva. }\end{array}$ & $\begin{array}{l}\text { STROBE: } \\
21 / 22 \\
\text { SJR: Q2 }\end{array}$ \\
\hline $\begin{array}{l}\text { Min } Y \text {, et } \\
a^{R^{7}} \text {. China, } \\
2021 .\end{array}$ & $\begin{array}{l}\text { Estudio } \\
\text { observacional } \\
\text { retrospectivo. }\end{array}$ & 74 & $\begin{array}{l}\text { Comparar las } \\
\text { diferencias en las } \\
\text { características } \\
\text { epidemiológicas } \\
\text { y clínicas entre } \\
\text { pacientes que } \\
\text { sobrevivieron con } \\
\text { CoVID-19 y los que } \\
\text { fallecieron. }\end{array}$ & HD & $\begin{array}{l}\text { La mitad de los fallecidos eran mayores de } \\
66 \text { años y las comorbilidades principales eran } \\
\text { diabetes e hipertensión. Las características } \\
\text { basales de ambos grupos (supervivientes y } \\
\text { fallecidos) eran similares. }\end{array}$ & $\begin{array}{l}\text { STROBE: } \\
21 / 22 \\
\text { SJR: Q2 }\end{array}$ \\
\hline $\begin{array}{l}\text { Kakkanattu } \\
\text { TJ, et a }{ }^{R 8} \text {. } \\
\text { India, } 2021\end{array}$ & $\begin{array}{l}\text { Estudio } \\
\text { observacional } \\
\text { retrospectivo. }\end{array}$ & 1.273 & $\begin{array}{l}\text { Informar sobre } \\
\text { los resultados de } \\
\text { la infección por } \\
\text { COVID-19 en } \\
\text { pacientes de centros } \\
\text { de HD. }\end{array}$ & HD & $\begin{array}{l}\text { Los resultados fueron: } \\
\text { - Incidencia de } 8,7 \% \text {. } \\
\text { - } 98,6 \% \text { hospitalizados. } \\
\text { - Mortalidad } 22,9 \% \text { relacionada con mayor } \\
\text { edad, comorbilidades y mayor antigüedad } \\
\text { recibiendo diálisis. }\end{array}$ & $\begin{array}{l}\text { STROBE: } \\
\text { 19/22 } \\
\text { SJR: Q1 }\end{array}$ \\
\hline $\begin{array}{l}\text { Corbett RW, } \\
\text { et a }{ }^{29} \text {. Rei- } \\
\text { no Unido, } \\
2020 .\end{array}$ & $\begin{array}{l}\text { Estudio } \\
\text { observacional } \\
\text { de cohortes } \\
\text { prospectivo. }\end{array}$ & 300 & $\begin{array}{l}\text { Informar sobre el } \\
\text { efecto de la epidemia } \\
\text { en una población de } \\
\text { diálisis, identificando } \\
\text { los factores de } \\
\text { riesgo de contraer } \\
\text { la infección y las } \\
\text { características de la } \\
\text { transmisión. }\end{array}$ & $H D, D P$ & $\begin{array}{l}\text { Los análisis confirman una incidencia de } 19,6 \% \text {. } \\
\text { Se incorporó una rutina de cribado en cada sesión } \\
\text { de diálisis a cada paciente: tomar temperatura } \\
\text { e investigar sintomatología. Surgió la necesidad } \\
\text { de la diálisis ambulatoria aislada durante para } \\
\text { aquellos pacientes contagiados. }\end{array}$ & $\begin{array}{l}\text { STROBE: } \\
20 / 22 \\
\text { SJR: Q1 }\end{array}$ \\
\hline $\begin{array}{l}\text { Pizarro- } \\
\text { Sánchez } \\
\text { MS, et aßo. } \\
\text { España, } \\
2021 .\end{array}$ & $\begin{array}{l}\text { Estudio } \\
\text { observacional } \\
\text { retrospectivo. }\end{array}$ & 50 & $\begin{array}{l}\text { Analizar el espectro } \\
\text { clínico del COVID-19 } \\
\text { en pacientes en HD. }\end{array}$ & HD & $\begin{array}{l}\text { Los datos demográficos de la población } \\
\text { fueron: edad media de } 71 \text { años y presencia de } \\
\text { hipertensión, diabetes y ECV. La sintomatología } \\
\text { común era disnea, fiebre, diarrea y una alta } \\
\text { prevalencia de neumonía }(68,4 \%) \text {. La mitad } \\
\text { de los pacientes fueron hospitalizados y el } 14 \% \\
\text { fallecieron. }\end{array}$ & $\begin{array}{l}\text { STROBE: } \\
21 / 22 \\
\text { SJR: Q2 }\end{array}$ \\
\hline $\begin{array}{l}\text { Flythe JE, } \\
\text { et a } a^{\beta 1} \text {. Esta- } \\
\text { dos Unidos, } \\
2020 .\end{array}$ & $\begin{array}{l}\text { Estudio } \\
\text { observacional } \\
\text { de cohortes } \\
\text { retrospectivo. }\end{array}$ & 143 & $\begin{array}{l}\text { Examinar la evolución } \\
\text { clínica de pacientes } \\
\text { críticos con COVID-19 } \\
\text { y enfermedad renal } \\
\text { preexistente. }\end{array}$ & $H D, D P$ & $\begin{array}{l}\text { El } 74 \% \text { de los pacientes necesitaron ventilación } \\
\text { mecánica invasiva y en } 28 \text { días habían fallecido } \\
\text { más del } 50 \% \text { de los pacientes críticos. Se asoció } \\
\text { la presencia de ERC en diálisis con un mayor } \\
\text { riesgo de mortalidad. }\end{array}$ & $\begin{array}{l}\text { STROBE: } \\
22 / 22 \\
\text { SJR: Q1 }\end{array}$ \\
\hline $\begin{array}{l}\text { Kastali M et } \\
a^{\beta^{2}} \text {. Argelia, } \\
2020 .\end{array}$ & $\begin{array}{l}\text { Estudio } \\
\text { observacional } \\
\text { prospectivo. }\end{array}$ & 70 & $\begin{array}{l}\text { Mejorar la } \\
\text { comprensión de los } \\
\text { aspectos diagnósticos, } \\
\text { terapéuticos y } \\
\text { pronósticos de esta } \\
\text { enfermedad. }\end{array}$ & $H D, D P$ & $\begin{array}{l}\text { La complicación más frecuente fue la } \\
\text { desaturación oxígeno < } 70 \% \text { (ingreso en UCI). } \\
\text { La mortalidad estaba relacionada con: presencia } \\
\text { de diabetes, disnea y consciencia alterada al } \\
\text { diagnóstico y necesidad de ingreso en UCI. }\end{array}$ & $\begin{array}{l}\text { STROBE: } \\
\text { 17/22 } \\
\text { SJR: Q3 }\end{array}$ \\
\hline
\end{tabular}




\begin{tabular}{|c|c|c|c|c|c|c|}
\hline Autor & Tipo de estudio & Muestra & Objetivos & $\begin{array}{l}\text { Tipo } \\
\text { de diálisis }\end{array}$ & Conclusiones & $\begin{array}{l}\text { Evaluación } \\
\text { de calidad }\end{array}$ \\
\hline $\begin{array}{l}\text { Hilbrands } \\
\text { LB, et } a^{\beta 3} \text {. } \\
\text { Reino Uni- } \\
\text { do, } 2020 \text {. }\end{array}$ & $\begin{array}{l}\text { Estudio } \\
\text { observacional } \\
\text { de cohortes } \\
\text { prospectivo. }\end{array}$ & 768 & $\begin{array}{l}\text { Presentar las } \\
\text { consecuencias } \\
\text { y asociar las } \\
\text { características } \\
\text { del paciente, la } \\
\text { enfermedad y el } \\
\text { tratamiento con la } \\
\text { mortalidad. }\end{array}$ & $H D, D P$ & $\begin{array}{l}\text { Los pronósticos fueron: } 70 \% \text { hospitalizados, } 12 \% \\
\text { ingresaron en UCI y una mortalidad del } 25 \% \text {. Los } \\
\text { factores de riesgo asociados a mortalidad: mayor } \\
\text { edad, debilidad, presencia de obesidad, síntomas } \\
\text { de disnea y fiebre alta, como los más importantes. }\end{array}$ & $\begin{array}{l}\text { STROBE: } \\
22 / 22 \\
\text { SJR: Q1 }\end{array}$ \\
\hline $\begin{array}{l}\text { Zou R et } \\
\text { a } \beta^{4} \text {. China, } \\
2020 .\end{array}$ & $\begin{array}{l}\text { Estudio } \\
\text { observacional } \\
\text { de cohortes } \\
\text { retrospectivo. }\end{array}$ & 66 & $\begin{array}{l}\text { Informar de las } \\
\text { características clínicas } \\
\text { y las consecuencias } \\
\text { de pacientes en HD } \\
\text { con infección por } \\
\text { COVID-19. }\end{array}$ & HD & $\begin{array}{l}\text { Las complicaciones más frecuentes fueron: } \\
\text { SDRA, fallo respiratorio, lesión cardiaca aguda, } \\
\text { fallo cardiaco y shock. Se asoció la mortalidad } \\
\text { con los siguientes factores de riesgo: disnea y } \\
\text { fiebre, enfermedades coronarias o alteraciones } \\
\text { analíticas prominentes. }\end{array}$ & $\begin{array}{l}\text { STROBE: } \\
21 / 22 \\
\text { SJR: Q2 }\end{array}$ \\
\hline $\begin{array}{l}\text { Valeri MA, } \\
\text { et a }{ }^{\beta 5} \text {. } \\
\text { Nueva York, } \\
2020 \text {. }\end{array}$ & $\begin{array}{l}\text { Estudio } \\
\text { observacional } \\
\text { de cohortes } \\
\text { retrospectivo. }\end{array}$ & 59 & $\begin{array}{l}\text { Examinar la } \\
\text { presentación, } \\
\text { evolución clínica, } \\
\text { consecuencias y } \\
\text { factores de riesgo } \\
\text { de enfermedad más } \\
\text { grave en pacientes con } \\
\text { ERC hospitalizados } \\
\text { por COVID-19. }\end{array}$ & $H D, P D$ & $\begin{array}{l}\text { Entre los síntomas de la enfermedad, el } 8 \% \text { de } \\
\text { los pacientes presentaron una alteración del } \\
\text { estado mental. La mortalidad se asociaba a } \\
\text { mayor edad y a un índice de comorbilidad de } \\
\text { Charlson incrementado. }\end{array}$ & $\begin{array}{l}\text { STROBE: } \\
\text { 19/22 } \\
\text { SJR: Q1 }\end{array}$ \\
\hline $\begin{array}{l}\text { Seidel } M \text { et } \\
a^{\beta 6} . \text { Alema- } \\
\text { nia, } 2020 .\end{array}$ & $\begin{array}{l}\text { Estudio } \\
\text { observacional } \\
\text { retrospectivo. }\end{array}$ & 56 & $\begin{array}{l}\text { Informar sobre } \\
\text { caracterización clínica } \\
\text { de COVID-19 en } \\
\text { pacientes en HD e } \\
\text { identificar factores } \\
\text { de riesgo de mal } \\
\text { pronóstico. }\end{array}$ & HD & $\begin{array}{l}\text { El curso de la enfermedad se dividió en: } \\
\text { enfermedad leve, grave (hospitalización), crítica } \\
\text { (UCI) y letal. La mortalidad se asocia con mayor } \\
\text { edad y con la presencia de síndrome cardiorrenal. }\end{array}$ & $\begin{array}{l}\text { STROBE: } \\
\text { 19/22 } \\
\text { SJR: Q1 }\end{array}$ \\
\hline $\begin{array}{l}\text { Roper T et } \\
\text { a }{ }^{\beta 7} \text {. Reino } \\
\text { Unido, } \\
2020 .\end{array}$ & $\begin{array}{l}\text { Estudio } \\
\text { observacional } \\
\text { retrospectivo. }\end{array}$ & 76 & $\begin{array}{l}\text { Reflejar la efectividad } \\
\text { de las medidas } \\
\text { tomadas para reducir } \\
\text { las transmisión viral } \\
\text { mientras se ofrece } \\
\text { una óptima HD a los } \\
\text { pacientes. }\end{array}$ & HD & $\begin{array}{l}\text { La mortalidad se asoció a pacientes de } 77 \text { años } \\
\text { de media, con mayor número de comorbilidades y } \\
\text { de debilidad clínica. Se implementaron medidas } \\
\text { para frenar la transmisión y estrategias para } \\
\text { optimizar los cuidados. Además, surgió un modelo } \\
\text { de predicción de infección específico de diálisis } \\
\text { (SIR). }\end{array}$ & $\begin{array}{l}\text { STROBE: } \\
\text { 19/22 } \\
\text { SJR: Q1 }\end{array}$ \\
\hline $\begin{array}{l}\text { Lodge DM, } \\
\text { et } a^{\beta 8} \text {. Rei- } \\
\text { no Unido, } \\
2020 .\end{array}$ & $\begin{array}{l}\text { Estudio } \\
\text { observacional } \\
\text { prospectivo. }\end{array}$ & 166 & $\begin{array}{l}\text { Reducir la exposición } \\
\text { frente al COVID-19, } \\
\text { así como la } \\
\text { transmisión entre los } \\
\text { pacientes en HD. }\end{array}$ & HD & $\begin{array}{l}\text { Finalmente, } 113 \text { pacientes se adaptaron al } \\
\text { cambio de protocolo, y el resto tuvieron que } \\
\text { ser devueltos a las } 3 \text { sesiones semanales de } \\
\text { diálisis. Se redujeron } 452 \text { exposiciones al virus al } \\
\text { disminuir las visitas al centro sanitario. }\end{array}$ & $\begin{array}{l}\text { STROBE: } \\
\text { 20/22 } \\
\text { SJR: Q2 }\end{array}$ \\
\hline $\begin{array}{l}\text { Apata IW, } \\
\text { et a }{ }^{\beta 9} \text {. Esta- } \\
\text { dos Unidos, } \\
2021 .\end{array}$ & $\begin{array}{l}\text { Estudio } \\
\text { observacional } \\
\text { prospectivo. }\end{array}$ & 751 & $\begin{array}{l}\text { Describir las medidas } \\
\text { de control de la } \\
\text { infección COVID-19 } \\
\text { implementadas y las } \\
\text { características clínicas } \\
\text { de los pacientes } \\
\text { contagiados. }\end{array}$ & $H D, D P$ & $\begin{array}{l}\text { Las actividades para el control de la infección se } \\
\text { implementaron en tres fases: } \\
\text { - Procedimientos de cribado, clasificación y } \\
\text { separación de los pacientes con síntomas. } \\
\text { - Medidas adicionales como telemedicina. } \\
\text { - Manejo de pacientes confirmados con } \\
\text { COVID-19 en la unidad de diálisis. }\end{array}$ & $\begin{array}{l}\text { STROBE: } \\
\text { 19/22 } \\
\text { SJR: Q2 }\end{array}$ \\
\hline
\end{tabular}

\section{Evolución clínica de la enfermedad}

Un estudio clasificó el curso de la enfermedad en cuatro estadios: curso leve con buen control ambulatorio, curso grave con hospitalización del paciente o curso crítico de la enfermedad con ingreso en una Unidad de Cuidados Intensivos $(\mathrm{UCI})^{36}$.
Se analizaron 8 artículos para determinar el curso clínico de la enfermedad y los resultados fueron:

- Alrededor del $40 \%$ de los pacientes pasaron por una enfermedad leve controlada desde su domicilio, según dos artículos revisados ${ }^{18,25}$. 
- La tasa de hospitalización era superior al 50\% en la mayoría de los artículos analizados 17,18,20,23,25,30,33,36.

Por lo tanto, la presentación grave de la enfermedad se presentó al menos en la mitad de la población de estudio. La principal causa de hospitalización y enfermedad grave fue la hipoxia ${ }^{17}$. Además, se relacionó la hospitalización con pacientes de mayor edad, fumadores, con arritmias o con dependencia ${ }^{25}$.

- La evolución crítica que suponía el ingreso en UCI fue muy variable en los distintos estudios analizados: rango entre $5 \%$ y $23,6 \%{ }^{24,25}$. En un estudio, el $16 \%$ de los pacientes en estado crítico no fue trasladado a UCI debido a un pronóstico demasiado malo ${ }^{32}$. El motivo más frecuente de presentación crítica de la enfermedad fue el agravamiento de la disnea ${ }^{21,24}$, que desencadenaba en la aplicación de ventilación mecánica invasiva en un gran porcentaje de los pacientes ingresados en UCI (rango entre $68-100 \%)^{23,24,33,35}$.

\section{- Mortalidad, causas y factores de riesgo}

La tasa de mortalidad en este grupo de pacientes ascendía a más del $20 \%$ en la mayoría de los estudios incluidos que analizaban este dato (rango entre 20\% y $31 \%)^{17,18,20-28,33-36}$. En un artículo que incluía solo pacientes en estado crítico ingresados en $\mathrm{UCI}^{31}$, la tasa de mortalidad en los primeros 28 días de enfermedad llegó a superar el $50 \%$.

Las complicaciones más frecuentes por COVID-19 que llevaron al fallecimiento de los pacientes fueron: causas pulmonares (fallo respiratorio secundario a SDRA), causas cardíacas (lesión cardíaca agua y fallo cardíaco), shock séptico, CID (coagulación intravascular diseminada), daño hepático agudo o hemorragias (por ejemplo: gastrointestinales) $)^{18,20,34}$.

Los factores de riesgo de mortalidad más mencionados en los artículos revisados son:

- Edad avanzada. La edad mayor a 65 años es un potente predictor de mortalidad ${ }^{17,23}$, aunque la mayor tasa de fallecimientos por COVID-19 en la población en diálisis se registra en el grupo de edad de entre 75-80 años 22,25 .

- Multimorbilidad ${ }^{17,18,25,27,28}$. Las comorbilidades como la hipertensión y la diabetes se relacionaban con aumento del riesgo. Las enfermedades cardiovasculares también contribuían a un gran aumento del riesgo de mortalidad, en concreto: arteriopatía coro- naria, insuficiencia cardíaca, cardiopatía isquémica o síndrome cardiorrenal (en un estudio fallecieron más del $80 \%$ de los que lo padecían) ${ }^{36}$. También aumentaba el riesgo de fallecimiento un mayor índice de comorbilidad de Charlson ${ }^{35}$.

- Fragilidad o debilidad clínica 25,33,37. Se determinaba por marcadores como la hipoalbuminemia o la incapacidad para caminar ${ }^{25}$, 0 a través de la escala CFS (Clinical Frailty Score), con una puntuación igual 0 superior a cinco $^{33}$.

- Sexo masculino ${ }^{22}$. Se identificó como factor de riesgo en varios estudios en los que la proporción de hombres fallecidos superaban el $70 \%$ del total de la población ${ }^{28,36}$.

- Presentación crítica de la enfermedad. Tanto el diagnóstico de neumonía ${ }^{17}$ como el ingreso en $\mathrm{UCI}^{32}$ y la utilización de ventilación mecánica ${ }^{24}$ (más presentes en el grupo de fallecidos) son factores que determinan una mayor mortalidad. Otro factor predictor de presentación crítica y mortalidad es un cociente PF $\left(\mathrm{PaO}_{2} / \mathrm{FiO}_{2}\right)$ disminuido, que se traduce en insuficiencia respiratoria aguda 26 .

- Medidas de prevención y control en unidades de HD. Los centros sanitarios de HD comenzaron a aplicar medidas de prevención y control para minimizar la transmisión y frenar la expansión del virus. Estas medidas se basaron en 19,29,37,39:

- Proceso rutinario de detección a la entrada de los pacientes en el centro para realizar su sesión de diálisis. Este proceso se basaba en: toma de temperatura, cribado de síntomas y de exposición/contacto con personas infectadas con COVID-19. En un estudio ${ }^{19}$ incluían en el cribado la realización de tomografías computarizadas pulmonares y análisis de sangre en los pacientes de hemodiálisis.

- Los pacientes que resultaban sospechosos de infección por COVID-19 se sometían a una prueba nasofaríngea. Si resultaban positivos, eran aislados durante la sesión de diálisis en unidades u hospitales habilitados únicamente para pacientes contagiados. Además, en pacientes sospechosos o confirmados se usaba el transporte individual en lugar de colectivo para acudir al centro ${ }^{37}$.

- Se instauró el uso obligatorio de mascarilla quirúrgica por parte de todos los pacientes y uso de equipos de protección reforzados (mascarilla FFP2/ 
N95, uso de bata y protectores faciales) para los profesionales sanitarios.

- Aplicación de la telemedicina. Se basaba en reuniones virtuales mensuales a través de una aplicación de videoconferencias para los pacientes de diálisis domiciliaria (pacientes de DP y de HD domiciliaria). Se realizaban de forma multidimensional, incluyendo al paciente, el dietista, el trabajador social, la enfermera y el médico39.

- Una universidad de Londres (Imperial College of London) diseñó un modelo de predicción SIR (sospechoso-infectado-recuperado) específico para diálisis. Fue utilizado en uno de los estudios para predecir y planificar la positividad por COVID-19 en la población de HD ${ }^{37}$.

Además de prevención y control, también se aplicaron medidas para optimizar la atención a los pacientes: aumento de la presencia del médico en la unidad de HD COVID-19-positiva para supervisar los parámetros; realizar dos sesiones semanales de HD en los pacientes nuevos; y continuar con 3 sesiones semanales de HD en los pacientes actuales ${ }^{37}$.

En relación con este último aspecto, se analizó un estudio llevado a cabo en Reino Unido que tenía como objetivo reducir de forma segura la frecuencia de la HD de tres sesiones semanales a dos por semana para disminuir la exposición de los pacientes y la transmisión del COVID-19. El 68\% de la población incluida en el estudio fueron capaces de continuar con dos sesiones semanales de HD, lo que se tradujo en 452 exposiciones potenciales menos al COVID-19 durante la diálisis o el transporte a los centros ${ }^{38}$.

\section{Discusión}

Tras revisar todos los artículos incluidos en la revisión bibliográfica, se ha podido evidenciar que la incidencia de infección por COVID-19 en la población de estudio varía en función del tipo de diálisis que se lleva a cabo. La incidencia fue mayor en pacientes en HD que los que recibían diálisis en el domicilio, ya sea peritoneal o HD domiciliaria ${ }^{20}$. Estos resultados están respaldados por dos artículos realizados en unidades de diálisis domiciliaria ${ }^{40}$ y HD hospitalaria ${ }^{41}$, en los que la incidencia de coronavirus fue del $15,1 \%$ y del $41,1 \%$, respectivamente. Las unidades de HD son lugares de especial consideración porque los pacientes deben acudir al centro sanitario para el tratamiento y se exponen a diversos factores: transporte sanitario colectivo, reunión en la unidad en la que reciben el tratamiento y contacto con el personal sanitario $0^{42}$. Por el contrario, los pacientes en DP reciben el tratamiento en sus domicilios y no se exponen a tales riesgos, siendo esta vertiente del tratamiento dialítico un factor protector frente a la infección de SARS-CoV-2.

La sintomatología reflejada en los distintos estudios de la población en diálisis se solapa con la comunicada en la población general ${ }^{6}$, aunque uno de los artículos confirma que las alteraciones del estado mental eran más prevalentes en población de HD que en la población sin ERC $(25 \% \text { vs. } 12 \%)^{31}$. Los pacientes en diálisis tienen vulnerabilidad neurológica relacionada con la enfermedad vascular y, además, la isquemia inducida por el tratamiento de diálisis o los cambios osmolares los hacen susceptibles a los efectos neurológicos de COVID-19 (directos e indirectos, como estrés oxidativo, hipoxia o isquemia $)^{43,44}$.

Tal y como describen nuestros resultados, en la población de diálisis fue muy frecuente que la enfermedad COVID-19 se presentara de forma moderada/grave y que los pacientes tuvieran que ser hospitalizados ${ }^{17}$. En la población general, los datos de la evolución clínica difieren bastante con los de la población en diálisis: solo el $15 \%$ (frente el $50 \%$ en la población dializada) fueron hospitalizados por síntomas más graves, el $5 \%$ fueron ingresados en una UCI y el $80 \%$ de los casos fue una enfermedad leve que podía confundirse con un resfriado o gripe ${ }^{45}$.

Con respecto a la mortalidad, los resultados fueron muy elevados ya que, en los estudios analizados, la tasa de mortalidad se encontraba entre el $20 \%$ y el $31 \%$. En la población general española, según los datos del Ministerio de Sanidad, la tasa de mortalidad alcanzó el $2,2 \%{ }^{46}$. En definitiva, tras analizar estos dos últimos aspectos, podemos inferir que la enfermedad cursa con mayor gravedad y causa más muertes en la población en diálisis que en la población general. La causa de estas diferencias radica en los factores de riesgo que este tipo de pacientes poseen y que aumentan la presencia de dichos aspectos.

Los factores de riesgo más mencionados en la población con ERC en tratamiento con diálisis son la edad avanzada y la coexistencia de distintas enfermedades, como son la hipertensión, la diabetes o las enfermedades cardiovasculares, lo que aumenta su mortalidad respecto a la población general ${ }^{25}$. Sin embargo, según Martos Pérez 
et al. ${ }^{47}$, los factores de riesgo, respecto a la COVID-19, son similares en ambas poblaciones: edad de 65 años o mayor e hipertensión, diabetes o cardiopatía como la enfermedad cardiovascular más frecuente. Con estos datos, se determina que los enfermos renales, en concreto los que se encuentran en tratamiento con diálisis (HD o DP) son personas de riesgo frente a COVID-19 no por el hecho de padecer enfermedad renal de forma aislada, sino debido a la cantidad de enfermedades que subyacen a esta.

Otros factores de riesgo mencionados en los artículos evaluados son la debilidad clínica o el sexo masculino ${ }^{33,36}$. La debilidad clínica es un estado que se asocia con el envejecimiento y aumenta la vulnerabilidad frente a efectos adversos, como puede ser contraer una enfermedad, y se manifiesta con mayor morbilidad y mortalidad $^{48}$. Dado el perfil clínico de los pacientes en diálisis ${ }^{12,13}$, se pueden relacionar con cierta debilidad clínica. El sexo masculino se determinó como factor de riesgo en varios de los estudios de la revisión, aunque también destaca como factor de riesgo en estudios sobre población general. Esta relación se asocia a que las hormonas sexuales masculinas, en concreto la testosterona, aumenta la expresión de la enzima convertidora de angiotensina 2 (ECA 2) que está presente en la membrana de las células de varios sistemas (cardiovascular, riñones, intestino, etcétera). A su vez, el SARS-CoV-2 tiende a unirse a los receptores de ECA 2, estableciendo así el mecanismo de presentación grave y de riesgo de muerte de la enfermedad ${ }^{49}$.

Por otro lado, las medidas de prevención y control de la infección fueron implementadas en todos los centros sanitarios, sociosanitarios u hospitales al inicio de la pandemia. En concreto, las unidades de HD españolas se basaron en un documento técnico que el Ministerio de Sanidad propuso sobre las medidas necesarias para frenar el COVID-19 y consensuó con las Sociedades Científicas, médica y de enfermería. Este documento, al igual que los artículos analizados, incluía medidas de prevención y control a través del cribado de pacientes a la llegada al centro, la protección e información sanitaria a los profesionales, recomendación de uso de transporte propio o limitar el colectivo a cuatro personas $y$, manejo y ubicación adecuado del paciente con COVID-1950.

\section{Limitaciones del estudio}

Las principales limitaciones de este estudio han sido el escaso tiempo revisado, circunstancia impuesta por la realidad de aparición del coronavirus responsable de la COVID-19 a principios de 2020; lo que ha condicionado la falta de homogeneidad en los estudios $y$, por tanto, en los resultados, lo que ha dificultado el modo de estructurar los mismos.

\section{Consideraciones prácticas}

Consideramos que esta revisión puede ayudar a los profesionales de enfermería en su práctica diaria a gestionar mejor el manejo del paciente COVID-19 en las unidades de diálisis, porque a pesar del conocimiento adquirido en el último año y los programas de vacunación, quedan muchas incertidumbres sobre el SARS-CoV-2 y, sobre todo, mucho tiempo de convivencia con este virus. Por tanto, todo el conocimiento científico que ayude a cuidar mejor a los pacientes en diálisis desde la perspectiva de la prevención de las infecciones, y a los profesionales para su propia prevención, será indispensable en el futuro.

A la vista de los resultados extraídos de esta revisión, podemos resumir como principales conclusiones:

- La incidencia de la COVID-19 en las unidades de HD es mayor a la de las técnicas domiciliarias, como la DP, debido a la necesidad del paciente de acudir al centro sanitario y todo lo que conlleva la estancia en él. Por otro lado, las características clínicas que la COVID-19 provoca en los pacientes de diálisis son similares a las de la población general, aunque la proporción de casos que cursan más graves con ingreso en UCI es mayor.

- La alta mortalidad en los pacientes en diálisis se debe a sus antecedentes personales y médicos, tales como la edad avanzada y las comorbilidades asociadas a su patología renal. Por tanto, presentan los mismos factores de riesgo de mortalidad que la población general.

- En cuanto a las medidas de control en las unidades de HD, existe un protocolo de actuación, que la mayoría de los centros sanitarios acataron al inicio de la pandemia; como el uso de equipos de protección individual o cribado de los pacientes a su llegada a las unidades de diálisis. Adicionalmente, se han utilizado otras medidas para prevenir el riesgo de contagio como la telemedicina, la reducción de las sesiones de HD o la individualización del transporte sanitario.

Recepción: 12-09-21

Aceptación: 09-11-21

Publicación: 30-12-21 


\section{Bibliografía}

1. Zhu N, Zhang D, Wang W, Li X, Yang B, Song J, et al. A Novel Coronavirus from Patients with Pneumonia in China, 2019. N Engl J Med. 2020;382:727-33.

2. Lu R, Zhao X, Li J, Niu P, Yang B, Wu H, et al. Genomic characterisation and epidemiology of 2019 novel coronavirus: implications for virus origins and receptor binding. Lancet. 2020;395(10224):56574.

3. COVID-19: Events as they happen. WHO (World Health Organization). [Consultado 2 Mar 2021]. Disponible en: https://www.who.int/emergencies/ diseases/novel-coronavirus-2019/events-as-they-happen.

4. Coronaviridae Study Group of the International Committee on Taxonomy of Viruses. The species severe acute respiratory syndrome-related coronavirus: classifying 2019-nCoV and naming it SARSCoV-2. Nat Microbiol. 2020;5:536-44.

5. Feng Z, Li Q, Zhang Y, Wu Z, Dong X, Ma H, et al. The epidemiological characteristics of an outbreak of 2019 Novel Coronavirus Diseases (COVID-19)-China, 2020. CCDC Weekly. 2020;2(8):113-22.

6. Guan W, Ni Z, Hu Y, Liang W, Ou C, He J, et al. Clinical Characteristics of Coronavirus Disease 2019 in China. N Engl J Med. 2020;382(18):1708-20.

7. Zheng $Y$, Xu H, Yang M, Zeng $Y$, Chen $H$, Liu R, et al. Epidemiological characteristics and clinical features of 32 critical and 67 noncritical cases of $\mathrm{CO}$ VID-19 in Chengdu. J Clin Virol. 2020;127.

8. Eknoyan G, Lameire N, Barsoum R, Eckardt K, Levin $A$, Levin $N$, et al. The burden of kidney disease: improving global outcomes. Kidney Int. 2004;66:1310-4.

9. Otero A, de FA, Gayoso P, García F. Prevalence of chronic renal disease in Spain: results of the EPIRCE study. Nefrología. 2010;30(1):78-86.

10. Kidney Diseases: Improving Clinical Outcomes (KDIG0) CKD Work Group. KDIG0 2012 Clinical Practice Guideline for the Evaluation and Management of Chronic Kidney Disease. Kidney Int Suppl.
2013;3:1-150. Disponible en: https://kdigo.org/ wp-content/uploads/2017/02/KDIG0_2012_CKD_ GL.pdf.

11. National Institute of Diabetes and Digestive and Kidney Diseases. Health Information: Insuficiencia Renal. [consultado 04 Mar 2021]. Disponible en: https://www.niddk.nih.gov/health-information/informacion-de-la-salud/enfermedades-rinones/insuficiencia-renal.

12. Registro Español de Enfermos Renales (REER). Informe de diálisis y trasplante 2019. [consultado 06 Abr 2021]. Disponible en: https://www.senefro.org/contents/webstructure/INFORME_REER SEN_2020_WEB_SEN.pdf.

13. Gutiérrez D, Leiva-Santos J, Macías MJ, Cuesta A. Perfil sintomático de los pacientes con Enfermedad Renal Crónica Estadio 4 y 5 . Enferm Nefrol. 2017;20(3):259-66.

14. Urrutia G, Bonfill X. Declaración PRISMA: una propuesta para mejorar la publicación de revisiones sistemáticas y metaanálisis. Med Clin. 2010;135(11):507-11.

15. Vandenbroucke J, Von E, Altman D, Gøtzsche $P$, Mulrow C, Pocock S, et al. Mejorar la comunicación de estudios observacionales en epidemiología (STROBE): explicación y elaboración. Gac Sanit. 2009;23(2):1-28.

16. Scimago Journal \& Country Rank. [consultado May 2021]. Disponible en: https://www.scimagojr.com/.

17. Anoun M, Khalil R, Mahfoud W, Fatfat $H$, Khalil $\mathrm{L}$, Alameddine $\mathrm{R}$, et al. Age and multimorbidities as por prognostic factors for COVID-19 in hemodialysis: a Lebanese national study. BMC Nephrol. 2021;22(73).

18. Alberici F, Delbarba E, Manenti $C$, Econimo L, Valerio $F$, Pola $A$, et al. A report from the Brescia Renal COVID Task Force on the clinical characteristics and short-term outcome of hemodialysis patients with SARS-CoV-2 infection. Kidney Int. 2020;98:20-6.

19. Xiong F, Tang H, Liu L, Tu C, Tian J, Lei C, et al. Clinical Characteristics of and Medical Interventions for COVID-19 in Hemodialysis Patients in Wuhan, China. J Am Soc Nephrol. 2020;31:1387-97. 
20. Hsu C, Weiner D, Aweh G, Miskulin D, Manley H, Stewart C, et al. COVID-19 Among US Dialysis Patients: Risk Factors and Outcomes from a National Dialysis Provider. Am J Kidney Dis. 2021;77(5):748-56.

21. Lano G, Braconnier A, Bataille S, Cavaille G, Moussi-Frances J, Gondouin B, et al. Risk factors for severity of COVID-19 in chronic dialysis patients from a multicentre French cohort. Clin Kid J. 2020;13(4): 878-88.

22. Jager K, Kramer A, Chesnaye N, Couchoud C, Sánchez-Álvarez J, Garneata L, et al. Results from the ERA-EDTA Registry indicate a high mortality due to COVID-19 in dialysis patients and kidney transplant recipients across Europe. Kidney Int. 2020;98:1540-8.

23. Taji $L$, Thomas $D$, Oliver $M$, Ip J, Tang $Y$, Yeung $A$, et al. COVID-19 un patients undergoing long-term dialysis in Ontario. Can Med Assoc J. 2021;193(8):278-84.

24. Turgutalp K, Ozturk S, Arici M, Eren N, Gorgulu N, Islam $\mathrm{M}$, et al. Determinants of mortality in a large group of hemodialysis patients hospitalized for $\mathrm{CO}$ VID-19. BMC Nephrol. 2021;22(29).

25. Couchoud C, Bayer F, Ayav C, Béchade C, Brunet $F$, Chantrel $F$, et al. Low incidence of SARS-CoV-2, risk factors of mortality and the course of illness in the French national cohort of dialysis patients. Kidney Int. 2020;98(6):1519-29.

26. Tomacruz DI, So PN, Pasilan RM, Camenforte JK, Duavit MI. Clinical Characteristics and Short-Term Outcomes of Chronic Dialysis Patients Admitted for COVID-19 in Metro Manila, Philippines. Int J Nephrol Renovasc Dis. 2021;14:41-51.

27. Min $Y$, Cheng L, Tu C, Li H, He D, Huang D, et al. Clinical characteristics of deceased hemodialysis patients affected by COVID-19. Int Urol Nephrol. 2021;53:797-802.

28. Kakkanattu TJ, Sankarasubbaiyan S, Yadav AK, Kundu M, Gowda N, Kumar V, et al. Outcome and Determinants of Outcome of COVID-19 Infection Among Hemodialysis Patients: Findings from a National Dialysis Network Program in India. Kidney Int Rep. 2021;6(5):1429-32.
29. Corbett RW, Blakey S, Nitsch D, Loucaidou M, McLean A, Duncan N, et al. Epidemiology of COVID-19 in an Urban Dialysis Center. J Am Soc Nephrol. 2020;31(8):1815-23.

30. Pizarro-Sánchez MS, Avello A, Mas-Fontao S, Stock da Cunha T, Goma-Garcés E, Pereira $M$, et al. Clinical Features of Asymptomatic SARS-CoV-2 Infection in Hemodialysis Patients. Kidney Blood Press Res. 2021;46:126-34.

31. Flythe JE, Assimon MM, Tugman MJ, Chang EH, Gupta S, Shah J, et al. Characteristics and Outcomes of Individuals with Pre-existing Kidney Disease and COVID-19 Admitted to Intensive Care Units in the United States. Am J Kidney Dis. 2020;77(2):190203.

32. Kastali M, Kada AY, Ounnas S. Impact of COVID-19 infections on hemodialysis patients in the province of Blida, Argelia. Pan Afr Med J. 2020;31(1). [consultado 04 Abr 2021]. Disponible en: https://www. ncbi.nlm.nih.gov/pmc/articles/PMC7934889/pdf/ PAMJ-SUPP-37-1-51.pdf.

33. Hilbrands LB, Duivenvoorden $R$, Vart $P$, Franssen $C$, Hemmelder $\mathrm{MH}$, Jager KJ, et al. COVID-19-related mortality in kidney transplant and dialysis patients: results of the ERACODA collaboration. Nephrol Dial Transplant. 2020;35(11):1973-83.

34. Zou R, Chen F, Chen D, Xu C, Xiong F. Clinical characteristics and outcome of hemodialysis patients with COVID-19: a large cohort study in a single Chinese center. Ren fail. 2020;42(1):950-7.

35. Valeri AM, Robbins-Juarez SY, Stevens JS, Ahn W, Rao MK, Radhakrishnan J, et al. Presentations and Outcomes of Patients with ESKD and COVID-19. J Am Soc Nephrol. 2020;31(7):1409-15.

36. Seidel M, Hölzer B, Appel H, Babel N, Westhoff TH. Impact of renal disease and comorbidities on mortality in hemodialysis patients with COVID-19: a multicenter experience from Germany. J Nephrol. 2020;31:871-4.

37. Roper T, Kumar N, Lewis-Morris T, Moxham V, Kassimatis T, Game D, et al. Delivering Dialysis During the COVID-19 Outbreak: Strategies and Outcomes. Kidney Int Rep. 2020;5(7):1090-4. 
38. Da Silva $M$, Abeygunaratne $T$, Alderson $H$, Ali $I$, Brown N, Chrysochou C, et al. Safely reducing hemodialysis frequency during the COVID-19 pandemic. BMC Nephrol. 2020;21(52).

39. Apata IW, Cobb J, Navarrete J, Burkart J, Plantinga $L$, Lea JP. COVID-19 infection control measures and outcomes in urban dialysis centers in predominantly African American communities. BMC Nephrol. 2021;22(81).

40. Maldonado M, Ossorio M, Del Peso G, Santos C, Álvarez $L$, Sánchez-Villanueva $R$, et al. Incidencia y resultados de la COVID-19 en una unidad de diálisis domiciliaria en Madrid (España) durante el pico de la pandemia. Nefrología. 2020;42(3):329-36.

41. Albalate $M$, Arribas $P$, Torres $E$, Cintra $M$, Alcázar $\mathrm{R}$, Puerta $\mathrm{M}$, et al. Alta prevalencia de COVID-19 asintomático en hemodiálisis. Aprendiendo día a día el primer mes de pandemia de COVID-19. Nefrología. 2020;40 (3):279-86.

42. Arenas MD, Villar J, González C, Cao H, Collado $\mathrm{S}$, Crespo $\mathrm{M}$, et al. Manejo de la epidemia por coronavirus SARS-CoV-2 (COVID-19) en unidades de hemodiálisis. Nefrología. 2020;40(2):258-64.

43. Varatharaj A, Thomas N, Ellul M, Davies N, Pollak T, Tenorio $E$, et al. Neurological and neuropsychiatric complications of COVID-19 in 153 patients: a UK-wide surveillance study. Lancet. 2020;7(10):875-82.

44. Nuzzo D, Picone P. Potential neurological effects of severe COVID-19 infection. Neurosci. Res. 2020;158:1-5.
45. Paules $C$, Marston $H$, Fauci A. Coronavirus Infections-More than just the Common Cold. JAMA. 2020;323(8):707-8.

46. Ministerio de Sanidad. Actualización $n^{\circ} 367$. Enfermedad por el coronavirus (COVID-19). Situación en España. [consultado 4 May 2021]. Disponible en: https://www.mscbs.gob.es/profesionales/saludPublica/ccayes/alertasActual/nCov/documentos/Actualizacion_367_COVID-19.pdf.

47. Martos $F$, Luque J, Jiménez N, Mora E, Asencio C, García JM, et al. Comorbilidad y factores pronósticos al ingreso en una cohorte COVID-19 de un hospital general. Rev Clin Esp. 2020.

48. Fried L, Tangen C, Walston J, Newman A, Hirsch C, Gottdiener J, et al. Frailty in older adults: evidence for a phenotype. J Gerontol. 2001;56(3):146-56.

49. Gutiérrez MF, Juárez OC. Obesidad y sexo masculino como factores de riesgo para casos moderados a severos de COVID-19. Rev Cienc Tecnol. 2021;17(1):19-23.

50. Ministerio de Sanidad. Recomendaciones para el manejo, prevención y control de COVID-19 en Unidades de Diálisis. [consultado 2 Abr 2021]. Disponible en: https://www.mscbs.gob.es/profesionales/salud Publica/ccayes/alertasActual/nCov/documentos/COVID19-hemodialisis.pdf.

Este artículo se distribuye bajo una Licencia Creative Commons Atribución-NoComercial 4.0 Internacional. https://creativecommons.org/licenses/by-nc/4.0/ 